\title{
Ruptured Left Sinus of Valsalva Aneurysm
}

\author{
Manphool Singhal, Divyesh Mahajan, Rajiv Mahajan, Niranjan Khandelwal
}

\section{ABSTRACT}

The left sinus of valsalva aneurysm (SVA) is a very uncommon condition which can compress the coronary arteries leading to potential catastrophic complications. We present a case of a contained rupture of left SVA causing significant mass effect on the adjacent cardiac chambers and coronary arteries diagnosed on computed tomographic angiography (CTA).

Keywords: Computed tomographic angiography, Sinus of valsalva aneurysm.

How to cite this article: Singhal M, Mahajan D, Mahajan R, Khandelwal N. Ruptured Left Sinus of Valsalva Aneurysm. J Postgrad Med Edu Res 2012;46(3):155-156.

Source of support: Nil

Conflict of interest: None declared

\section{INTRODUCTION}

Sinus of valsalva aneurysm (SVA) is a rare condition. In 75 to $90 \%$ of cases, right sinus of valsalva is involved, in $10 \%$ the noncoronary sinus is affected and the left sinus is very rarely affected. ${ }^{1}$ SVA are usually of two types-congenital and acquired mostly due to syphilis, atherosclerosis or endocarditis. ${ }^{2,3}$

Transesophageal echocardiography (TEE) and thoracic aortography are traditionally used for diagnosis and procedure planning. But TEE is operator dependent and thoracic aortography only gives an idea about vascular anatomy. There are very few cases of unruptured left SVA diagnosed on computed tomographic angiography (CTA). In this case, CTA not only accurately diagnosed the condition but also helped in timely intervention and planning of the surgery.

\section{CASE REPORT}

A 67-year-old man presented with a 1 week history of chest pain with mild dyspnea. The chest pain had increased in last 2 days. The patient was nonsmoker, nonalcoholic but had a 10-year history of hypertension.

On admission, physical examination and laboratory data, including cardiac enzyme levels and electrocardiogram (ECG) revealed no abnormalities. The chest radiograph revealed mild cardiomegaly. The patient underwent transthoracic echocardiography which revealed dilated left ventricle and dilated left sinus of valsalva.

For further characterization of the lesion, CTA was done which revealed $8 \times 7.4 \mathrm{~cm}$ partially thrombosed unruptured
SVA arising from the left sinus of valsalva (Fig. 1). It was causing significant mass effect on the left ventricle and aorta outflow tract. The right coronary artery was unremarkable, however the left anterior descending artery (LAD) and left circumflex (LCX) were stretched and compressed around the hematoma (LAD > LCX; Fig. 2). The patient was operated and doing well.

\section{DISCUSSION}

Complicated SVA should be managed timely and aggressively as delay in intervention can lead to increased morbidity and mortality. Majority of series have shown increased incidence of the right sinus of valsalva involvement with rupture into the right ventricular outflow tract, right ventricle and right atrium in diminishing incidence respectively. ${ }^{4}$ Left SVA rupture is rare. ${ }^{4,5}$ Left SVA usually rupture into the pulmonary artery, the left ventricle, the myocardium or even the epicardium. ${ }^{6}$ Unruptured valsalva sinus aneurysms may lead to complications depending on their size and mass effect on adjacent cardiac structures. ${ }^{7}$

TEE is usually the first imaging modality for evaluation of the cardiac function and status of the major vessels and cardiac chambers. It is safe, noninvasive and easily available but it is operator dependent and complete evaluation may not be possible in certain patients due to technical difficulties. Further imaging may be needed to confirm the diagnosis. TEE offers the potential for more accurate characterization of the aneurysm. ${ }^{8}$

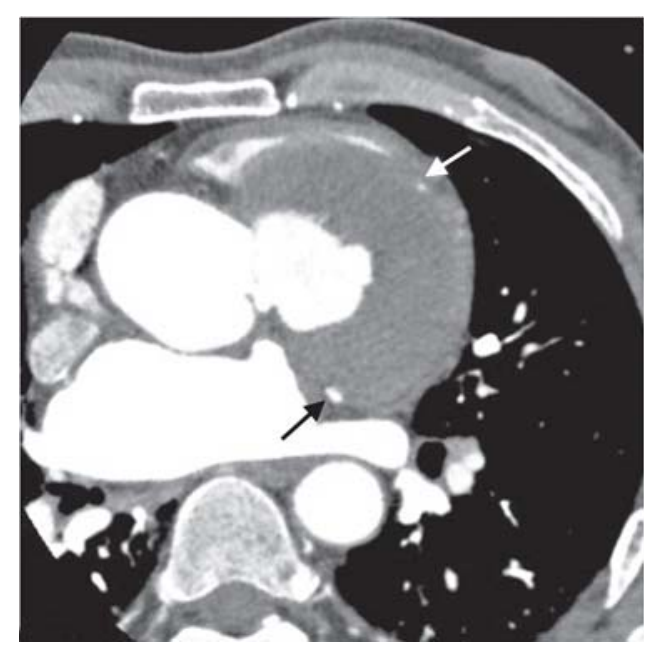

Fig. 1: Axial CT image shows ruptured left sinus of valsalva with a large peripheral hypodense hematoma. The LAD (white arrow) and LCX (black arrow) are seen splayed around the hematoma with attenuated calibers 


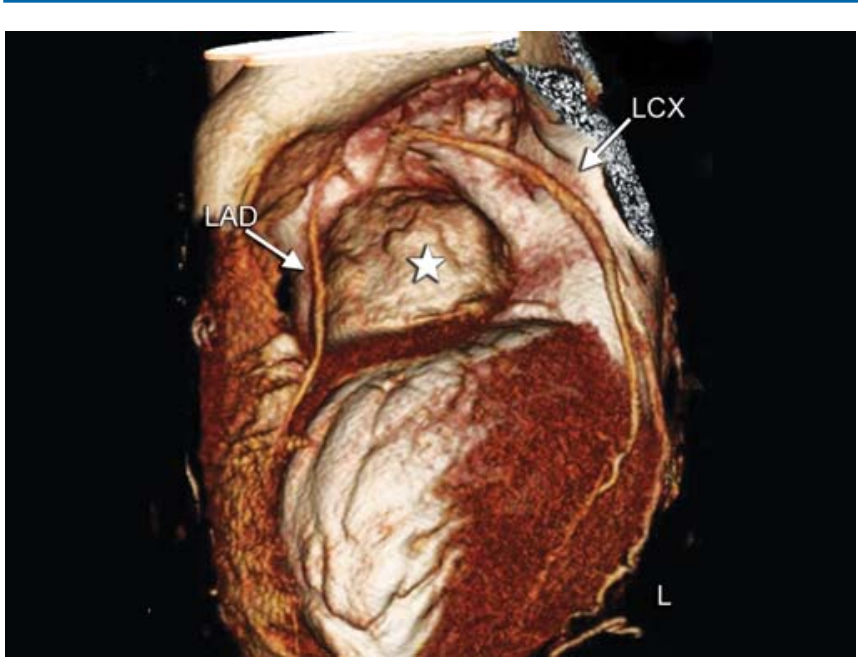

Fig. 2: Volume rendered images demonstrate contrast leak (asterisk) from left sinus splaying the LAD and LCX (LCX > LAD)

CTA has advantage over invasive coronary angiography as detail of the cardiac chambers is provided along with vascular detail. In addition, there is no risk of injury to the aortic root containing a ruptured sinus and pseudoaneurysm by catheter manipulation. The volume-rendered images (VRT), maximum intensity projection (MIP) and curved reformatted images (CPR) provide a precise anatomical details which help in surgical planning as done in our case.

\section{REFERENCES}

1. Goldberg N, Krasnow N. Sinus of valsalva aneurysms. Clin Cardiol 1990;13:831-36.

2. Guo DW, Cheng TO, Lin ML, Gu ZQ. Aneurysm of the sinus of valsalva: A roentgenologic study of 105 Chinese patients. Am Heart J 1987;114:1169-77.

3. Edwards JE, Burchell HB. The pathological anatomy of deficiencies between the aortic root and the heart, including aortic sinus aneurysms. Thorax 1957;12:125-39.
4. Shah RP, Ding ZP, Ng A, Quek A. Ten-year review of ruptured sinus of valsalva: Clinicopathological and echo-Doppler features. Singapore Med J 2001;42:473-76.

5. Park SH, Jung HS, Yu M, Min SK, Ahn JH, Kim Y. Left valsalva sinus aneurysm rupture into left atrium and aortic valve prolapse confirmed with transesophageal echocardiography. J Am Soc Echocardiogr 2007;20:1010.e3-e6.

6. Chu SH, Hung CR, How SS, Chang $\mathrm{H}$, Wang SS, Tsai $\mathrm{CH}$, et al. Ruptured aneurysms of the sinus of valsalva in oriental patients. J Thorac Cardiovasc Surg 1990;99:288-98.

7. Aliye Ozsoyoglu BrickerBindu AvutuTan-Lucien H, Mohammed Eric E, et al. Valsalva sinus aneurysms: Findings at CT and MR Imaging. Radio Graphics 2010;30:99-110.

8. Wang KY, St John Sutton M, Ho HY, Ting CT. Congenital sinus of valsalva aneurysm: A multiplane transesophageal echocardiographic experience. J Am Soc Echocardiogr 1997;10: 956-63.

\section{ABOUT THE AUTHORS}

\section{Manphool Singhal (Corresponding Author)}

Assistant Professor, Department of Radiodiagnosis, Postgraduate Institute of Medical Education and Research, Chandigarh160012, India, Phone: +91-1722756381, Fax: +91-1722745768 e-mail: drmsinghal@yahoo.com

\section{Divyesh Mahajan}

Assistant Professor, Department of Radiodiagnosis, Postgraduate Institute of Medical Education and Research, Chandigarh, India

\section{Rajiv Mahajan}

Assistant Professor, Department of Cardiology, Postgraduate Institute of Medical Education and Research, Chandigarh, India

\section{Niranjan Khandelwal}

Professor and Head, Department of Radiodiagnosis, Postgraduate Institute of Medical Education and Research, Chandigarh, India 